\title{
LEY MATEMÁTICA DE LOS SISTEMAS DINÁMICOS CARDIACOS: REDUCCIÓN DE SU TIEMPO DE
} EVALUACIÓN A 18 HORAS

MATHEMATICAL LAW OF CARDIAC DYNAMIC SYSTEMS: REDUCTION OF ITS EVALUATION TIME TO

\section{HOURS \\ LEI MATEMÁTICA DOS SISTEMAS CARDÍACOS DINÂMICOS: REDUÇÃO DO TEMPO DE AVALIAÇÃO \\ PARA 18 HORAS}

Javier Oswaldo Rodríguez Velásquez'; Sandra Catalina Correa Herrera ${ }^{2}$; Alejandro Pizano ${ }^{3}$; Miguel Alberto Ronderos ${ }^{4}$; Signed Esperanza Prieto Bohórquez ; Carol Godoy ${ }^{6}$; Juan Alexander Rojas ${ }^{7}$; Juan Benitez ${ }^{8}$; Dharma Rodríguez Correa ${ }^{9}$ Eliana

Avilan $^{10}$

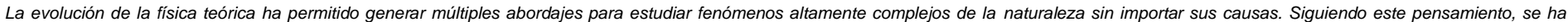

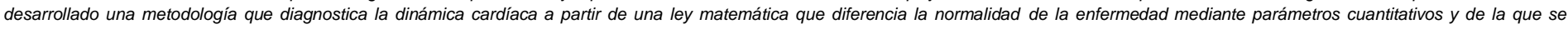
demuestra su aplicabilidad clínica al reducir su tiempo de aplicación.

Conceptos claves:

Se corroboró la capacidad para diagnosticar la dinámica cardiaca en 18 horas de la ley caótica exponencial y, se demostró su facultad predictiva de forma objetiva y reproducible.

\section{Resumen:}

Introducción: Anteriormente se desarrolló una ley físico-matemática para la evaluación de registros electrocardiográficos continuos y Holters con la cual se dedujo la totalidad atractores cardíacos y se diferenció normalidad, estados patológicos y evolución entre estados. Método: Fueron tomadas 200 dinámicas cardiacas, 150 con diferentes tipos de patologías cardiacas y 50 normales, a las cuales se aplicó la ley exponencial, en 18 y 21 horas. Para ello, se simuló una secuencia de frecuencias cardiacas, con la cual fue construido el atractor caótico. A continuación, se determinó el diagnóstico matemático a partir de la ley, con base en la ocupación espacial del atractor y se calculó la dimensión fractal. Finalmente, se realizó la validación estadística del método matemático en 18 horas frente al Gold Standard. Resultados: Sujetos con dinámicas cardiacas caóticas normales presentaron valores en la rejilla Kp entre 205 y 384 , mientras que los sujetos con dinámicas patológicas presentaron valores entre 61 y 191 en 18 horas. La evaluación de la concordancia entre el diagnóstico matemático en 18 horas y la evaluación convencional tomada Gold Standard, dio como resultado valores de sensibilidad y especificidad de $100 \%$ y un coeficiente Kappa de 1. Conclusión: Se confirmó la capacidad clínica de la ley para diagnosticar de forma objetiva y reproducible en 18 horas.

Palabras clave: fractales; sistemas no lineales; diagnóstico; electrocardiografía ambulatoria

\section{Abstract:}

Introduction: Previously, a physical-mathematical law was developed for the evaluation of continuous electrocardiographic and Holter registers, with which all cardiac attractors were deduced and normality, pathological states and evolution between states were differentiated. Method: There were taken 200 cardiac dynamics, 150 with different types of cardiac pathologies and 50 normal ones, to which the exponential law was applied in 18 and 21 hours. For this, a sequence of heart rates was simulated, with which the chaotic attractor was constructed. Next, the mathematical diagnosis was determined with the law based on the spatial occupation of the attractor, and the fractal dimension was calculated. Finally, statistical validation of the mathematical method in 18 hours was performed against the Gold Standard. Results: Subjects with normal chaotic cardiac dynamics presented values in the Kp grid between 205 and 384, whereas subjects with pathological dynamics presented values between 61 and 191 in 18 hours. The evaluation of the concordance between the mathematical diagnosis in 18 hours and the conventional evaluation, taken as Gold Standard resulted in sensitivity and specificity values of $100 \%$ and a Kappa coefficient of 1. Conclusion: It was confirmed the clinical capacity of the law to diagnose objectively and with reproducibility in 18 hours.

Keywords: fractals; non-linear systems; diagnosis; ambulatory electrocardiography

\section{Resumo}

Introducción: uma lei físico-matemática foi desenvolvida para a avaliação de registros eletrocardiográficos contínuos e Holters com os quais todos os atratores cardíacos foram deduzidos e normalidade, estados patológicos e evolução entre os estados foram diferenciados. Método: foram tomadas 200 dinâmicas cardíacas, 150 com diferentes tipos de patologias cardíacas e 50 normais, às quais foi aplicada a lei exponencial, em 18 e 21 horas. Para isso, foi simulada uma sequência de frequências cardíacas, com a qual foi construído o atrator caótico. Em seguida, o diagnóstico matemático foi determinado a partir da lei, com base na ocupação espacial do atrator e calculada a dimensão fractal. Por fim, a validação estatística do método matemático foi realizada em 18 horas contra o Gold Standard. Resultados: Sujeitos com dinâmica cardíaca caótica normal apresentaram valores na grade de Kp entre 205 e 384, enquanto sujeitos com dinâmica patológica apresentaram valores entre 61 e 191 em 18 horas. A avaliação da concordância entre o diagnóstico matemático em 18 horas e a avaliação convencional feita pelo Gold Standard, resultou em valores de sensibilidade e especificidade de 100\% e coeficiente Kappa de 1. Conclusão: A capacidade clínica da lei para diagnosticar de forma objetiva e reprodutível em 18 horas foi confirmada. 


\section{INTRODUCCIÓN}

El estudio de la dinámica de los sistemas, se realiza mediante la teoría de los sistemas dinámicos ${ }^{1}$. Una forma de análisis de la evolución de sus variables dinámicas es su representación geométrica en espacios conocidos como espacios de fase, donde se grafican valores de una o más variables consecutivamente en el tiempo. Estas representaciones geométricas son denominadas atractores, y permiten establecer, entre otras cosas, si la dinámica es predecible, en cuyo caso adquieren una forma puntual o cíclica, o impredecible, en cuyo caso se obtiene un atractor caótico. Los atractores caóticos se caracterizan por su forma irregular, razón por la cual son cuantificados mediante geometría fractal, desarrollada por Mandelbrot $^{2-4}$. Existen diferentes métodos para la obtención de la dimensión fractal, definidos a partir de las características del objeto irregular en cuestión; los fractales salvajes son evaluados con el método de Box Counting ${ }^{5,6}$ a su vez, los fractales estadísticos son cuantificados mediante la ley de Zipf-Mandelbrot ${ }^{7,8}$.

La principal causa de muerte a nivel mundial son las Enfermedades Cardiovasculares; su incidencia presenta diferencias muy significativas en las distintas regiones a nivel mundial, siendo los países de ingresos bajos y medios los más afectados. La tasa de mortalidad por ésta causa ascienden al $30 \%$ del total de muertes en el mundo, de las cuales casi el $50 \%$ se asocian a Infarto Agudo de Miocardio ${ }^{9}$. Esta situación hace de este problema una de las prioridades de salud pública a nivel mundial y requiere el desarrollo de mejoras en los métodos de prevención, detección temprana y diagnóstico oportuno de los individuos ${ }^{9}$, especialmente en patologías asociadas con situaciones de riesgo vital como la fibrilación auricular, la cual se relaciona con accidentes cerebrovasculares ${ }^{10,11}$.

En condiciones intrahospitalarias el seguimiento de pacientes en estado crítico se realiza mediante monitores que permiten el registro electrocardiográfico continuo de la dinámica cardiaca, mientras que a nivel ambulatorio se utiliza el Holter, mediante el cual se evalúa el comportamiento cardiaco durante un registro continuo de 24 a 48 horas, lo que ha hecho posible la detección de alteraciones a partir de la evaluación del segmento ST, el intervalo QT y los intervalos RR, entre otros, que son detectados mediante el electrocardiograma convencional1 ${ }^{12}$. Incluso con la información obtenida mediante estos registros aún existen dificultades para la consecución de diagnósticos de absoluta precisión y de pronósticos efectivos, que eviten desenlaces como la muerte cardiaca súbita. En este contexto, uno de los puntos de interés para la realización de estudios que den cuenta del comportamiento cardiaco en la actualidad es el análisis de la variabilidad de la frecuencia cardiaca (VFC) mediante los cambios $R R$ en el tiempo ${ }^{13,14}$. Sin embargo, a la fecha estos estudios son insuficientes para lograr aplicaciones generales a nivel clínico, haciendo necesaria nuevas formas de análisis de la información ${ }^{15-17}$.

Innovaciones desde los sistemas dinámicos han sido implementadas por Goldberger et al. ${ }^{16,17}$, quienes evidenciaron que la concepción homeostática tradicional no representaba de manera fidedigna el comportamiento real de la dinámica cardiaca, la cual se caracteriza por patrones altamente regulares o excesivamente irregulares en condiciones patológicas, mientras que la normalidad se establece como un estado intermedio entre estos extremos Rodríguez et al. ${ }^{18}$, han desarrollado nuevas metodologías desde teorías como la probabilidad o los sistemas dinámicos, logrando métodos diagnósticos de aplicabilidad clínica.

Recientemente, se desarrolló una ley matemática exponencial de los sistemas dinámicos cuya aplicación a la dinámica cardíaca en 21 horas permite establecer diferencias objetivas entre estados de normalidad y enfermedad aguda, así como la evolución entre estos estados, tanto en registros Holter como en electrocardiografía continua, cuantificando objetivamente el nivel de evolución de la enfermedad independientemente de su etiología o del número y frecuencia de alteraciones temporales. Su capacidad diagnóstica fue comprobada en estudios ciegos previamente realizados, incluyendo investigaciones llevadas a cabo en pacientes con diferentes tipos de $\operatorname{arritmias}^{19,20}$.

El propósito del presente estudio es realizar una aplicación de la ley exponencial en 18 horas con registros electrocardiográficos ambulatorios y continuos, en aras de confirmar su aplicabilidad clínica y su capacidad de establecer diagnósticos certeros en este lapso de tiempo.

\section{MÉTODOS}

\section{Definiciones}

Mapa de retardo: Tipo de atractor específico representado en un espacio abstracto de dos o más dimensiones, en donde se observa la dinámica de un sistema generada a partir de pares ordenados de valores consecutivos en el tiempo de una determinada variable dinámica.

Dimensión Fractal de Box-Counting:

$$
D=\frac{\log N\left(2^{-(K+1)}\right)-\log N\left(2^{-K}\right)}{\log 2^{k+1}-\log 2^{k}}=\log _{2} \frac{N\left(2^{-(k+1)}\right)}{N\left(2^{-k}\right)} \text { Ecuación } 1
$$

En donde $\mathrm{D}$ corresponde a la dimensión fractal; $\mathrm{N}$ corresponde al número de cuadros ocupado por el objeto $\mathrm{y}, \mathrm{k}$ corresponde al grado de partición de la cuadrícula. En este trabajo se utilizaron solo dos rejillas, denominadas $\mathrm{Kp}$ (cuadros pequeños) y Kg (cuadros grandes), así simplificando la expresión de la Ecuación 1, obtenemos:

$$
D=\log _{2} \frac{N\left(2^{-(k+l)}\right)}{N\left(2^{-k}\right)}=\log _{2} \frac{K_{p}}{K_{g}} \text { Ecuación } 2
$$

Ley matemática exponencial: Se realiza un despeje algebraico de la Ecuación 2, para dar origen a la ecuación con la cual se establece el diagnóstico físico-matemático, a saber:

$$
K_{p}=K_{g} 2^{D} \Rightarrow K_{g}=\frac{K_{p}}{2^{D}} \text { Ecuación 3 }
$$

Donde $\mathrm{Kp}$ son los espacios ocupados por el atractor en la rejilla pequeña. $\mathrm{Kg}$ son los espacios ocupados por el atractor en la rejilla grande y D es la Dimensión fractal.

\section{Población}

Se tomaron 200 trazados electrocardiográficos continuos y registros Holters con una duración mínima de 21 horas de registro, de individuos mayores de 21 años que fueron diagnosticados por un cardiólogo experto según los protocolos clínicos convencionales, 50 eran de pacientes diagnosticados como normales y 150 de ellos cursaban con patologías cardiacas. Los registros fueron tomados de bases de datos del Grupo Insight.

\section{Procedimiento}

Se enmascararon los resultados y diagnósticos clínicos de cada trazado electrocardiográfico. Luego, fueron tomados los valores máximos y mínimos de las frecuencias cardíacas y el número total de latidos por hora, durante 18 y 21 horas. Los anteriores valores fueron el punto de partida para la obtención del diagnóstico matemático. Mediante un software previamente desarrollado, se generó una secuencia de frecuencias cardiacas mediante un algoritmo equiprobable teniendo en cuenta los límites máximos y mínimos de cada registro. Estas secuencias fueron graficadas construyendo mapas de retardo (ver definiciones) 
A continuación, se calculó la dimensión fractal con el método de BoxCounting, superponiendo dos rejillas (Ecuación 2). La obtención de la dimensión fractal requirió el conteo de la ocupación espacial de los atractores en cada rejilla, valores que también fueron registrados para la obtención del diagnóstico matemático mediante la Ecuación 3 , a partir de los parámetros matemáticos establecidos en la aplicación en 21 horas de la ley ${ }^{20}$. De acuerdo con ésta, las dinámicas agudas presentan espacios de ocupación de Kp menores de 73, las dinámicas normales presentan espacios de ocupación mayores a 200 y el rango intermedio entre 73 y 200 , es considerado como evolución entre ambos estados. Se compararon los diagnósticos matemáticos obtenidos al aplicar esta metodología en los registros de las frecuencias cardiacas en 18 y 21 horas.

\section{Análisis estadístico}

Se desenmascararon los registros analizados y fue comparado el diagnóstico matemático en 18 y 21 horas para verificar la concordancia. Una vez demostrada, Se tomaron los casos normales y con enfermedad aguda en 18 horas para realizar una comparación con el diagnóstico médico convencional tomado como Gold Standard, determinando sensibilidad y especificidad en primera instancia.

Para esto, se realizó una clasificación binaria, en la que los verdaderos positivos (VP) representan los casos evaluados convencionalmente y matemáticamente como agudos; los falsos positivos (FP) representan los casos diagnosticados convencionalmente por parte del médico experto dentro de los límites de normalidad, que sin embargo presentaron valores matemáticos de agudización; los falsos negativos (FN) representan los casos que fueron evaluados matemáticamente dentro de límites normales pero que presentaron un diagnóstico convencional de agudización; por último, como verdaderos negativos (VN) se consideraron los casos diagnosticados convencionalmente y matemáticamente como normales.

Posteriormente, se evaluó la concordancia entre el diagnóstico convencional y el físico-matemático por medio del coeficiente Kappa, definido con la ecuación 4 :

$$
K=\frac{C o-C a}{T o-C a} \text { Ecuación } 4
$$

En donde, Co representa el número de concordancias observadas que corresponden al número de pacientes con el mismo diagnóstico desde la metodología matemática y desde el Gold Standard; To representa la totalidad de casos; $\mathrm{Ca}$ corresponde al número de concordancias atribuibles al azar que se calculan a través de la ecuación 5:

$$
C a=\left[\left(f_{1} x C_{1}\right) / T o\right]+\left[\left(f_{2} x C_{2}\right) / T o\right] \text { Ecuación } 5
$$

Donde f1 equivale al número de casos con valores matemáticos de normalidad; $\mathrm{C} 1$ representa el número de casos diagnosticados como normales por el experto clínico; f2 representa el número de casos evaluados matemáticamente como enfermedad; C2 representa el número de casos diagnosticados desde el ámbito clínico convencional con alguna patología; To representa el número total de casos.

\section{Aspectos éticos}

El presente estudio cumple con los principios éticos de la Declaración de Helsinki de la Asociación Médica Mundial y se establece como una investigación con riesgo mínimo, de acuerdo con la Resolución 8430 de 1993 del Ministerio de Salud de Colombia, en la medida que se realizan cálculos físicos y matemáticos sobre resultados de exámenes no invasivos que han sido prescritos previamente según protocolos médicos convencionales, sin interferir en ningún modo en su tratamiento médico, y protegiendo el anonimato e integridad de los pacientes. Ya que los datos provienen de bases de datos de investigaciones previas no fue necesario solicitar el consentimiento informado a los pacientes.

\section{RESULTADOS}

En la tabla 1 se observa el diagnóstico clínico de algunos de los registros evaluados. Se encontró que las dimensiones fractales de las dinámicas cardiacas evaluadas en 21 horas estuvieron entre 0,933 y 1,839 para los estados normales; y para los estados patológicos estuvieron entre 0,952 y 1,984. En 18 horas, se obtuvieron valores para las dimensiones fractales de los estados normales entre 0,906 y 1,847 ; y entre 1 y 1,961 para estados patológicos, evidenciando que no es posible establecer diferencias entre normalidad y enfermedad con la dimensión fractal. También, fue evidenciada concordancia en los diagnósticos matemáticos en 18 y 21 horas para el total de los casos.

Tabla 1. Información del diagnóstico clínico inicial de algunos de los registros electrocardiográficos continuos y ambulatorios seleccionados para el estudio. Valores de los espacios ocupados por los atractores cardiacos evaluados en 18 y 21 horas. Siendo $\mathrm{Kp}$, los valores de la rejilla de cuadros pequeños; $\mathrm{Kg}$ los valores de la rejilla de cuadros grandes y DF

\begin{tabular}{|c|c|c|c|c|c|c|c|}
\hline \multirow{2}{*}{ No. } & \multirow{2}{*}{ Diagnóstico clínico } & \multicolumn{3}{|c|}{21 horas } & \multicolumn{3}{|c|}{18 horas } \\
\hline & & $\mathrm{Kp}$ & $\mathrm{Kg}$ & DF & $\mathrm{Kp}$ & $\mathrm{Kg}$ & DF \\
\hline 1 & ECV en estudio & 188 & 59 & 1,672 & 188 & 57 & 1,722 \\
\hline 2 & Síncope en estudio & 184 & 79 & 1,220 & 184 & 77 & 1,257 \\
\hline 3 & Síncope en estudio & 192 & 61 & 1,654 & 197 & 63 & 1,645 \\
\hline 4 & Control médico & 110 & 31 & 1,827 & 114 & 32 & 1,833 \\
\hline 5 & Normal & 262 & 105 & 1,319 & 260 & 106 & 1,294 \\
\hline 6 & Bradicardia en estudio & 192 & 49 & 1,970 & 187 & 48 & 1,962 \\
\hline 7 & Normal & 229 & 64 & 1,839 & 234 & 65 & 1,848 \\
\hline 8 & Normal & 310 & 95 & 1,706 & 315 & 97 & 1,699 \\
\hline 9 & Normal & 258 & 73 & 1,821 & 259 & 72 & 1,847 \\
\hline 10 & Arritmia & 190 & 48 & 1,985 & 186 & 49 & 1,924 \\
\hline 11 & Sincope & 91 & 31 & 1,554 & 93 & 33 & 1,495 \\
\hline 12 & Normal & 204 & 67 & 1,606 & 207 & 68 & 1,606 \\
\hline 13 & $A C V$ & 91 & 36 & 1,338 & 95 & 38 & 1,322 \\
\hline$\overline{14}$ & Fibrilación auricular & 198 & 51 & 1,957 & 197 & 52 & $\overline{1,922}$ \\
\hline 15 & Normal & 363 & 123 & 1,561 & 362 & 125 & 1,534 \\
\hline 16 & $\begin{array}{l}\text { IAM, Ectopias ventriculares } \\
\text { infrecuentes, eventos de } \\
\text { trigeminismo, disminución de la } \\
\text { variabilidad de la frecuencia } \\
\text { cardiaca }\end{array}$ & 66 & 19 & 1,796 & 67 & 19 & 1,818 \\
\hline 17 & Normal & 209 & 76 & 1,459 & 205 & 75 & 1,451 \\
\hline 18 & Normal & 274 & 114 & 1,265 & 273 & 114 & 1,260 \\
\hline
\end{tabular}
la dimensión fractal. 


\begin{tabular}{|l|l|l|l|l|l|l|l|}
\hline 19 & Desvanecimiento & 115 & 33 & 1,801 & 112 & 35 & 1,678 \\
\hline 20 & Disfunción nodo sinusal & 92 & 27 & 1,769 & 87 & 27 & 1,688 \\
\hline 21 & Arritmia y dolor precordial en & & & & & & \\
& estudio & & & & & & \\
\hline 22 & Miocardiopatía hipertrófica & 60 & 31 & 0,953 & 64 & 32 & 1,000 \\
\hline 23 & Cardiopatía Isquémica en estudio & 166 & 52 & 1,675 & 163 & 50 & 1,705 \\
\hline 24 & Normal & & & & & & \\
\hline 25 & Normal & 361 & 189 & 0,934 & 358 & 191 & 0,906 \\
\hline 26 & Síncope en estudio & 209 & 80 & 1,385 & 213 & 79 & 1,431 \\
\hline 27 & Normal & 178 & 54 & 1,721 & 183 & 56 & 1,708 \\
\hline 28 & Normal & 309 & 156 & 0,986 & 309 & 156 & 0,986 \\
\hline 29 & Taquicardia en estudio & 388 & 118 & 1,717 & 384 & 117 & 1,715 \\
\hline 30 & Bradicardia en estudio & 177 & 50 & 1,824 & 181 & 50 & 1,856 \\
\hline 31 & Normal & 169 & 63 & 1,424 & 172 & 62 & 1,472 \\
\hline 32 & Sardiopatía isquémica, dislipidemia & 66 & 18 & 1,874 & 61 & 16 & 1,931 \\
\hline & & & & & & & \\
\hline
\end{tabular}

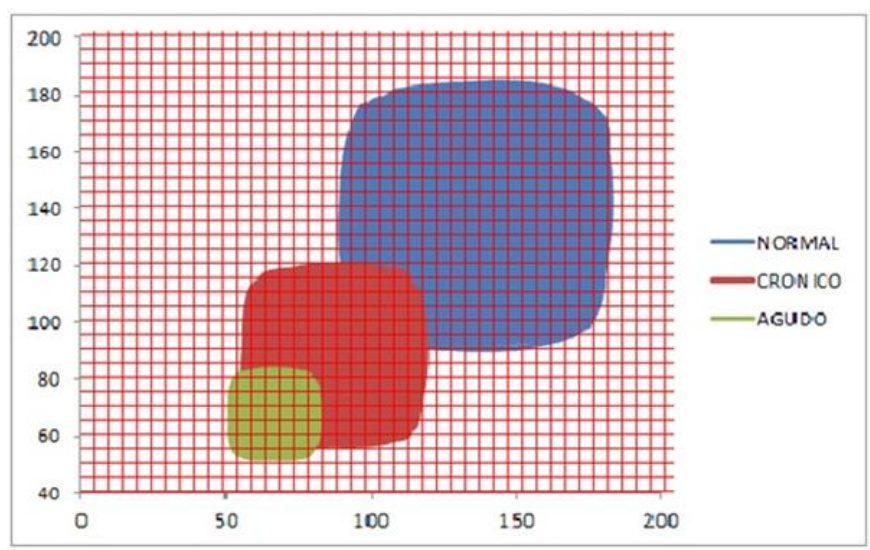

Figura 1. Atractores cardiacos en el espacio de Box Counting, en donde se pueden observar el comportamiento de una dinámica cardiaca normal, en evolución hacia la agudización y aguda. Los espacios de ocupación de estos atractores son para el caso agudo $\mathrm{Kp}=16$ y $\mathrm{Kg}=61$, para el estado evolutivo hacia enfermedad aguda $\mathrm{Kp}=54$ y $\mathrm{Kg}=199$; y para el caso normal $\mathrm{Kp}=381 \mathrm{y} \mathrm{Kg}=109$ (No 32, 33 y 31 de la Tabla 1, respectivamente).
En 21 horas se encontró que los espacios de ocupación en la rejilla $\mathrm{Kp}$ de las dinámicas patológicas estuvieron entre 60 y 198 y en la rejilla $\mathrm{Kg}$ se encontraron entre 18 y 79 . Para las dinámicas normales se encontraron valores en la rejilla Kp entre 204 y 388; y para la rejilla Kg entre 64 y 189 (ver tabla 1).

En 18 horas se obtuvieron valores para la rejilla Kp entre 205 y 384 , y para la rejilla $\mathrm{Kg}$ entre 65 y 91 para las dinámicas normales; así mismo, para estados patológicos se obtuvieron valores para la rejilla Kp entre 61 y 191 y para la rejilla Kg entre 16 y 77 (ver tabla 1). Los diagnósticos calculados coincidieron para cada caso de 21 y 18 horas. Por medio de los resultados se corroboró que los límites previamente establecidos para normalidad y enfermedad mediante la ley matemática aplicada a registros evaluados en 21 horas, también permite establecer diferencias en 18 horas por medio el análisis de la ocupación espacial de los atractores en la rejilla $\mathrm{Kp}$, esto fue corroborado con el análisis estadístico, con el cual se obtuvo sensibilidad y especificidad del $100 \%$ y coeficiente kappa igual a 1 tanto en 21 como en 18 horas.

En la figura 1 se muestran los atractores de una dinámica cardiaca normal, en evolución hacia la agudización y aguda; se evidencia que el espacio de ocupación del atractor de la dinámica normal es mucho mayor que el espacio de ocupación del atractor de la dinámica aguda; así mismo, se puede observar que el espacio de ocupación del atractor de la dinámica que evoluciona hacia enfermedad aguda se encuentra en un tamaño intermedio entre los atractores de las dos dinámicas anteriormente mencionadas.

\section{DISCUSIÓN}

Este es el primer trabajo en el que se confirma la capacidad diagnóstica y predictiva de una ley caótica exponencial de la dinámica cardiaca en 18 horas, a partir de un estudio ciego con 200 pacientes, logrando medidas de sensibilidad y especificidad del $100 \%$, y un coeficiente Kappa igual a 1, independientemente de la patología cardiaca estudiada, el sexo, edad, factores de riesgo, entre otros. Así mismo, se evidenció la capacidad de la metodología para cuantificar el nivel de gravedad del estado cardiaco a partir de la disminución espacial del atractor en el espacio de Box- Counting. Los resultados confirman que la dinámica cardiaca presenta un comportamiento no aleatorio, cuya autoorganización puede ser cuantificada de forma objetiva y reproducible desde la ley exponencial de los sistemas dinámicos cardiacos, siendo útil para el diagnóstico y seguimiento de pacientes, así como para la evaluación de la eficacia de intervenciones terapéuticas o farmacológicas.

Este trabajo evidencia que el uso de leyes matemáticas aplicadas al análisis de dinámicas fisiológicas cardiacas, proporciona una comprensión más profunda de sus características, y mejora los resultados logrados a partir del análisis de la variabilidad $R R$, dado que mientras que esta medida no permite establecer diferencias para cada caso particular y absoluta precisión al realizar comparaciones intra e interobservador, la ley aplicada arroja un resultado matemático específico para cada caso individual, al margen de consideraciones de tipo estadístico y recurriendo al orden matemático intrínseco del fenómeno.

Múltiples estudios desarrollados desde perspectivas no lineales, mediante la teoría del caos o el análisis fractal, han dado lugar a resultados prometedores y nuevas metodologías de análisis de estas dinámicas ${ }^{18,21-23}$. Tal es el caso de una metodología desarrollada por Huikuri et al. ${ }^{24}$, mediante la cual se establecen índices predictores de mortalidad en pacientes que han sufrido infarto agudo de miocardio y presentan una fracción de eyección bastante disminuida, mejorando los índices actuales, a partir de sistemas dinámicos y del uso simultáneo de medidas fractales. Este trabajo constituye un avance en este campo al estar fundamentado en una ley general que revela un orden que subyace a cualquier dinámica cardiaca, con implicaciones clínicas individuales, elevada objetividad y reproducibilidad, que además es susceptible de ser automatizado para su aplicación clínica a gran escala. 
La ley matemática aplicada en esta investigación se fundamenta en el razonamiento inductivo de la física teórica ${ }^{25}$, mediante la cual se establecen leyes y teorías físico-matemáticas, a partir del estudio de pocos casos representativos, para encontrar órdenes universales, aplicables a casos particulares. Este trabajo evidencia el carácter general de la ley, al ser aplicable en rangos de tiempo menores a los estudiados en la metodología inicial, desarrollada en 21 horas, evidenciando que es posible establecer afirmaciones objetivas y reproducibles en medicina a través de un pensamiento físicomatemático estricto, al margen de otras consideraciones.

Siguiendo esta forma de pensamiento se han encontrado diagnósticos y predicciones en fenómenos médicos como la hematología ${ }^{26}$, el proceso de reestenosis arterial ${ }^{27}$, la dinámica cardiaca del adulto ${ }^{28}$ y fetal $^{29}$, las alteraciones celulares en el desarrollo de cáncer de cuello uterino $^{30}$, la predicción de linfocitos T CD4 en pacientes infectados por el virus del $\mathrm{VIH}^{31}$, la predicciones de unión peptídica al HLA clase $\|^{32} \mathrm{o}$ la predicción de brotes de malaria en 820 municipios de Colombia ${ }^{33}$ e incluso predicciones de mortalidad ${ }^{34}$.

\section{Limitaciones de responsabilidad}

La responsabilidad del trabajo es exclusivamente de los autores.

\section{Conflictos de interés}

Ninguno

\section{Agradecimientos}

Agradecemos al Hospital Universitario Nacional de Colombia por su apoyo a nuestras investigaciones, especialmente al Doctor Giancarlo Buitrago, director del Instituto de Investigaciones Clínicas de la Universidad Nacional de Colombia-Hospital Universitario Nacional de Colombia.

\section{Originalidad del trabajo}

Este artículo es original y no ha sido enviado para su publicación a otro medio de difusión científica en forma completa ni parcialmente.

\section{Cesión de derechos}

Los participantes de este trabajo ceden el derecho de autor a la Universidad Nacional de Córdoba para publicar en la Revista de la Facultad de Ciencias Médicas y realizar las traducciones necesarias al idioma inglés.

\section{Participación de los autores}

JOR: Diseñó el estudio y creo la metodología;

AP: Apoyo en el análisis de los resultados matemáticos y revisión final del manuscrito.

MR: análisis de resultados desde ámbito clínico y redacto el borrador del manuscrito.

SCC y SEP: desarrollaron los cálculos matemáticos, el análisis de resultados y las correcciones finales del manuscrito.

CYG: análisis de resultados desde ámbito clínico y reviso el borrador del manuscrito.

JR y JB: análisis de resultados desde ámbito clínico.

DR y EA: recolección de datos y sistematización de los mismos.

\section{BIBLIOGRAFÍA}

1. Devaney, R. L., Siegel, P. B., Mallinckrodt, A. J., McKay, S. A first course in chaotic dynamical systems: theory and experiments. Computers in Physics 7.4 1993. 416-417.

2. Peitgen, H. O., Jürgens, H., Saupe, D. Chaos and fractals. Chaos and Fractals: New Frontiers of Science. New York: Springer-Verlag; 1992.

3. Mandelbrot $B$. The fractal geometry of nature. New York: $W$. H. Freeman and Company; 2000.

4. Mandelbrot B. ¿Cuánto mide la costa de Gran Bretaña? En: Mandelbrot B, editor. Los Objetos Fractales. Barcelona: Tusquets Eds. S.A.; 2000. 27- 50.

5. Peitgen $H$, Jürgens $H$, Saupe $D$, Forewords. En: Peitgen $H$, Jürgens $H$, Saupe $D$, editors.Chaos and Fractals: New Frontiers of Science. New York: Springer-Verlag. 1992:1- 7.

6. Peitgen $H$, Jurgens $H$, Saupe $D$. Lenght, area and dimension. Measuring complexity and scalling properties. En: Peitgen $H$, Jürgens $H$, Saupe $D$, editors. Chaos and Fractals:
New Frontiers of Science. New York: Springer-Verlag; 2004. $p$. 183-228.

7. Mandelbrot B. Árboles jerárquicos o de clasificación y la dimensión. En: Mandelbrot, editores. Los Objetos Fractales. Barcelona: Tusquets Eds. S.A.; 2000. p.161-66.

8. Burgos J. Zipf-scaling behavior in the immune system. Biosystems. 1996;39:227-32.

9. Organización Mundial de la Salud. [Internet] Enfermedades cardiovasculares; c2017[consultado el 25 de enero del 2019]. Centro de prensa.Disponible en:http://www.who.int/mediacentre/factsheets/fs317/es/index.html 10. Prieto S, Young P, Ceresetto JM, Bullorsky EO. Terapia anticoagulante en fibrilación auricular. Medicina. 2011;71(3):27482.

11. Neumar RW, Otto CW, Link MS, Kronick SL, Shuster M, Callaway CW, et al. Part 8: adult advanced cardiovascular life support: 2010 American Heart Association Guidelines for Cardiopulmonary Resuscitation and Emergency Cardiovascular Care. Circulation.2010 Nov 2;122(supp/3):S729-S767.

12. Al Rahhal M, Bazi $Y$, Al Hichri $H$, Alajlan $N$, Melgani $F$, Yager $R$. Deep learning approach for active classification of electrocardiogram signals.Information Sciences. $2013 \mathrm{Jul}$ 1;345:340-54.

13. Bayés A. Muerte súbita. Revista Española de cardiología. 2012 Nov; 65(11):1039-52.

14. Nolan J, Batin PD, Andrews R, Lindsay SJ, Brooksby $P$, Mullen $M$, et al. Prospective study of heart rate variability and mortality in chronic heart failure: results of the United Kingdom Heart Failure Evaluation and Assessment of Risk Trial (UK heart). Circulation. 1998 Oct 13; 98: 1510-6.

15. Walleczek J. Self-OrganizationBiological Dynamics and Nonlinear control.Cambridge, U.K: Cambridge Univ. Press;2000.

16. Goldberger A. Heartbeats, hormones, and Health - Is variability the spice of life? Am J Respir Crit Care Med. 2001May; 163(6): 1289-90.

17. Goldberger AL, Amaral L, Hausdorff J, Ivanov P, Peng CK, Stanley H. Fractal dynamics in physiology: Alterations with disease and aging. Proc Natl Acad Sci U S A. 2002 Feb 19; 99(Suppl 1): 2466-72.

18. Rodríguez J, Prieto P, Mendoza F, Pinilla L, Correa C, Soracipa $Y$, et al. Evaluación probabilista de la dinámica cardiaca arrítmica con y sin metoprolol. CES Med 2017; 31(2):144-154.

19. Rodríguez J, Narváez $R$, Prieto $S$, Correa $C$, Bernal $P$, Aguirre $G$, et al. The mathematical law of chaotic dynamics applied to cardiac arrhythmias. J. Med. Med. Sci. 2013 Jul;4(7):291-300.

20. Rodríguez J, Correa C, Melo M, Domínguez, D, Prieto S, Cardona DM, et al. Chaotic cardiac law: Developing predictions of clinical application. J. Med. Med. Sci. 2013 Feb;4(2): 79-84.

21. Garfinkel A. A mathematics for physiology. Am J Physiol. 1983 Oct;245(4): R455-66. doi: 0.1152/ajpregu.1983.245.4.R455. 22. Schumacher $A$. Linear and nonlinear approaches to the analysis of $R-R$ interval variability. Biol Res Nurs. 2004 Jan;5(3):211-21. doi: 10.1177/1099800403260619.

23. Scaffeta N, Moon R, West B. Fractal Response of Physiological Signals to Stress Conditions, Environmental Changes, and Neurodegenerative Diseases. Complexity. 2007 May 10;12(5):12-7.

24. Huikuri HV, Mäkikallio TH, Peng CK, Goldberger AL, Hintze $U$, Møller $M$. Fractal correlation properties of $R-R$ interval dynamics and mortality in patients with depressed left ventricular function after an acute myocardial infarction. Circulation. 2000 Jan 4-11;101(1):47-53. doi: 10.1161/01.cir.101.1.47

25. Einstein $A$. Sobre la teoría de la relatividad $y$ otras aportaciones científicas. Madrid: Sarpe; 1983. p. 23-32. 
26. Correa $C$, Rodríguez J, Prieto $S$, Álvarez L, Ospino $B$, Munévar $A$, et al. Geometric diagnosis of erythrocyte morphophysiology. J. Med. Med. Sci. 2012 Nov; 3(11): 715-20.

27. Rodríguez J, Prieto S, Correa C, Bernal P, Puerta G, Vitery $S$, et al. Theoretical generalization of normal and sick coronary arteries with fractal dimensions and the arterial intrinsic mathematical harmony. BMC Medical Physics. 2010; Sep;10:1.

28. Rodríguez J, Prieto S, Ramírez L. A novel heart rate atractor for the prediction of cardiovascular disease. Informatics in medicine. 2019; 15(100174):1-9.

29. Soracipa $Y$, Rodríguez J, Castillo M. Confirmación diagnóstica de la evaluación del trazado de la monitoria fetal apartir de la probabilidad y la relación $S / k$ de la entropía. Momento. 2018 Jul-Dic; 57:27-40.

30. Rodríguez J, Sánchez $M$, Barrios $F$, Soracipa $Y$. Geometrical Evaluation of Cervical Cells. Fractal and Euclidean Diagnostic Methodology of Clinical Application. Journal of Biosciences and Medicines. 2018 March;6:111-22.

31. Rodríguez J, Prieto S, Correa C, Melo M, Domínguez D, Olarte $N$, et al. Prediction refinement of CD4 cells number based on sets and probability theory. Current HIV Research. 2018; 16: 416-24.

32. Rodríguez J, Berna P, Prieto P, Correa C, Álvarez L, Pinilla $L$, et al. Predicción de unión de péptidos de Plasmodium falciparum al HLA clase II. Probabilidad, combinatoria y entropía aplicadas a las proteínas MSP-5 y MSP-6. Archivos de alergia e inmunología clínica. 2013 Jan;44(1):7-14.

33. Rodríguez J. Método para la predicción de la dinámica temporal de la malaria en los municipios de Colombia. Rev Panam Salud Pública. 2010;27(3):211-8.

34. Rodríguez J. Dynamical systems applied to dynamic variables of patients from the Intensive Care Unit (ICU). Physical and mathematical Mortality predictions on ICU.J.Med.Med. Sci. 2015 Nov; 6(8): 102-108. 